\title{
Filigrane
}

Écoutes psychothérapiques

\section{La langue : délaissée, rejetée, oubliée et retrouvée. Quelques réflexions, quelques questions}

\section{Carole Levaque}

Volume 18, numéro 2, automne 2009

Le corps. Sur le divan. Dans le fauteuil II

URI : https://id.erudit.org/iderudit/039289ar

DOI : https://doi.org/10.7202/039289ar

Aller au sommaire du numéro

Éditeur(s)

Revue Santé mentale au Québec

ISSN

1192-1412 (imprimé)

1911-4656 (numérique)

Découvrir la revue

Citer cet article

Levaque, C. (2009). La langue : délaissée, rejetée, oubliée et retrouvée. Quelques réflexions, quelques questions. Filigrane, 18(2), 51-69.

https://doi.org/10.7202/039289ar
Résumé de l'article

L'auteur décrit son " immigration » et son parcours linguistique ainsi que celui de ses patients. Elle s'intéresse à la violence de l'imposition de l'apprentissage d'une autre langue et fait des liens avec ce que subit le greffé : elle soulève des questions identitaires. Elle se questionne sur le refus d'apprendre une autre langue ou de la parler. Elle évoque le rejet de la langue maternelle et la rupture de liens qui accompagnent fréquemment ce rejet. Elle explore le rôle parfois défensif, parfois libérateur dans l'adoption d'une nouvelle langue. Elle termine en abordant quelques enjeux liés aux différences linguistiques du couple dans le processus thérapeutique. 


\title{
La langue: délaissée, rejetée, oubliée et retrouvée. Quelques réflexions, quelques questions
}

\author{
carole levaque
}

L'auteur décrit son «immigration» et son parcours linguistique ainsi que celui de ses patients. Elle s'intéresse à la violence de l'imposition de l'apprentissage d'une autre langue et fait des liens avec ce que subit le greffé: elle soulève des questions identitaires. Elle se questionne sur le refus d'apprendre une autre langue ou de la parler. Elle évoque le rejet de la langue maternelle et la rupture de liens qui accompagnent fréquemment ce rejet. Elle explore le rôle parfois défensif, parfois libérateur dans l'adoption d'une nouvelle langue. Elle termine en abordant quelques enjeux liés aux différences linguistiques du couple dans le processus thérapeutique.

The author describes her "immigration" and linguistic journey as well as that of her patients. She addresses the violence associated with the imposition of learning another language and links the process with organ transplants: she raises identity issues. She questions the refusal to learn another language or to speak it. The connections between the rejection of the mother tongue and the damaged relationships with the mother are highlighted. Both the defensive and the liberating aspects associated with the shift to a new language are explored through clinical vignettes. She concludes focusing on the impact of linguistic differences in the therapeutic couple and on the therapeutic process itself.

"Language is a twisted and dangerous thing, allied to the sweet and bloody violence of birth, the journey from the womb to the world, from the belly of the mother earth to the realm of the twists of language that constrain and bind."

(Kristina, J., "The Subject in process", in Edward Scheer, ed, Antoine Artaud, A Critical Reader, London and New York, 2004, 119)

"Pero las lenguas son sustancias radioactivas que hunden sus raíces en nuestro corazón más primitivo, en la oscura y irracional memoria de la horda. “

El País, España, Martes 8 de Julio 2008 (Lenguas, por Rosa Montero)

«Les langues diffèrent non pas par ce qu'elles peuvent ou non exprimer, mais par ce qu'elles nous obligent ou non à dire.»

(Hagège, C., dans Amati Mehler et al., La Babel de l'inconscient, Presses universitaires de France, 1994, 241) 
$\mathrm{L}$ 'écriture de ce texte est née du désir de comprendre et de partager mon parcours linguistique ainsi que celui de plusieurs de mes patients dont la majorité provient d'autres pays. Je m'intéresserai particulièrement à ceux à qui l'on a imposé l'apprentissage d'une autre langue, à ceux qui ont choisi d'adopter une troisième langue et enfin à ceux qui n'éprouvent pas le désir d'apprendre une autre langue malgré les avantages qu'ils en retireraient. J'explorerai l'acquisition d'une langue autre que la langue maternelle, la répétition de la violence originelle dans l'apprentissage d'une seconde langue, les motivations, les désirs, les non-désirs ainsi que certains des impacts identitaires non résolus ayant une influence sur cet apprentissage. J'aborderai également le rejet de la langue maternelle, les ruptures de liens fréquemment liées à ces rejets ainsi que l'expérience de renaissance et de libération parfois associée au choix d'une nouvelle langue.

\section{Mon «immigration»}

À l'âge de dix ans, «j'immigre» en Ontario, de l'autre coté de la rivière, à 12 kilomètres de la maison de mon enfance, située dans un petit village québécois. Ma mère, qui souhaitait que j'apprenne l'anglais, m'avait inscrite comme pensionnaire dans une école anglophone. Même si l'anglais ne m'était pas tout à fait étranger — c'était la langue secrète de mes parents, la langue qu'ils utilisaient lorsqu'ils ne voulaient pas que je les comprenne — je ne le parlais pas. Ce fut pour moi un moment de profonds changements. Je vécus une séparation pénible tout en ayant à composer avec une panoplie de nouvelles réalités extérieures. Cette transplantation de langue, de culture et de lieu provoquée par ma mère était une répétition de celle qu'elle avait vécue au même âge. Elle aussi avait dû traverser la rivière : prise en charge par un oncle curé qui l'installa au presbytère d'une petite ville ontarienne, elle fit ses études dans une école bilingue où l'on privilégiait l'anglais. L'apprentissage de l'anglais représentait pour ma mère une ouverture sur le monde et une chance de succès. Mais inconsciemment, je crois que cette rupture avec la langue de sa mère représentait aussi pour elle une autre façon de se définir, la possibilité d'adopter une identité autre que celle de sa propre mère.

L'apprentissage d'une autre langue entraîne un sentiment de nouveauté. Il permet d'entendre du jamais dit comme «yo te quiero» (je t'aime). Inconsciemment, l'acquisition d'une autre langue peut aussi servir à fuir les conflits originels ; des conflits qui se manifestent par des carences affectives, l'absence ou la fragilité de liens. La langue seconde permet une prise de distance émotionnelle par rapport aux mots de la langue d'origine, ces mots qui font partie du bain sonore et qui contiennent une charge émotive, sensorielle, mots liés au corps dans le cadre de la première relation.

À l'école que je fréquentais, je m'intéressais surtout aux étudiantes étrangères qui composaient une partie importante du corps étudiant. J'avais des affinités particulières avec les Sud-Américaines car elles aussi apprenaient l'anglais. Je n'étais pas la seule de laquelle on se moquait. Je prononçais «heir» (héritier) 
comme «hair» (cheveux) et devais passer plusieurs récréations à pratiquer la prononciation de «this and that» au lieu de «dis and dat». On riait aussi de ces Sud-Américaines et de leurs erreurs («cocodriles» au lieu de «crocodiles») À cette époque, j'aurais donc voulu apprendre l'espagnol, mais on m'imposa plutôt le latin. Ce n'est que beaucoup plus tard, à l'âge adulte, que je finis par satisfaire ce désir. Le choix d'une deuxième langue étrangère dans ce nouveau milieu résonnait peut-être avec mes propres sentiments d'étrangeté.

Peu à peu, mon français s'appauvrit tandis que mon anglais s'améliorait. Lorsque j'atteignis le niveau universitaire, l'anglais s'était définitivement installé dans ma vie extérieure et intérieure ; il y demeura d'ailleurs une grande partie de ma vie adulte. Ce changement entraîna un refus de parler ma langue maternelle, le français, refus qui se refléta par la suite chez mes enfants, qui le parlent aujourd'hui mais avec un accent anglais.

«Et si la langue mère se tait, et si une nouvelle langue remplace complètement la langue originaire... Quels sont les événements inconscients qui s'organiseront parallèlement par rapport à la relation avec l'image maternelle» (Amati Mehler et al., 1994, 73).

\section{La nouvelle langue, la greffe et l'identité}

Plusieurs liens peuvent être faits entre l'acquisition de la langue étrangère et le phénomène de la greffe. Le greffé est forcé de prendre et d'accepter à l'intérieur de lui-même quelque chose d'essentiel mais qui ne lui appartient pas, ce qui implique des risques de disfonctionnements, d'infections, de rejet, de pertes et même de mort. À ce propos, je trouve tout à fait pertinentes les réflexions de JeanLuc Nancy dans son texte intitulé L'Intrus (Éditions Galilée, 2000) :

«Très vite cependant, l'autre comme étranger peut se manifester. Cela se nomme le rejet; mon système immunitaire rejette celui de l'autre (Cela veut dire: j'ai deux systèmes, deux identités immunitaires).... Pour protéger le greffé la médecine abaisse son immunité pour qu'il puisse supporter l'étranger, parvenir à l'accepter. Il y a intrus en moi et je deviens étranger à moi-même. mais devenir étranger à moi ne me rapproche pas de l'intrus. Il semblerait plutôt que s'expose une loi générale de l'intrusion : il n'y a jamais eu une seule intrusion; dès qu'il s'en produit une, elle se multiplie, elle s'identifie dans ses différences internes renouvelées » (Nancy, 2000, 31)

La pulsion de vie de l'immigré l'incite à investir l'apprentissage d'une langue étrangère tout en le laissant avec un sentiment d'étrangeté et des changements identitaires. Le contre-investissement et la pulsion de vie seraient analogues aux médicaments immunitaires qui agissent en abaissant les réactions contre l'intrus. 
«L'apprentissage d'une ou de plusieurs langues doit compter avec des problèmes de séparation à un niveau interpersonnel comme au niveau d'une césure et d'une discrimination intra psychique» (Amati Mehler et al., 1994, 130)

L'imposition d'un changement de langue exige de la personne de s'adapter, de s'y faire, d'apprendre la langue qui deviendra la sienne, à laquelle elle s'identifiera, à tel point que parfois la langue qu'elle parlera ne sera plus la sienne et la sienne ne l'habitera plus de la même façon.

Les concepts qu'on associe avec l'identité tels l'introjection, l'incorporation, l'imitation et l'identification adhésive suscitent davantage de réflexion quand on explore des changements de langue. Nancy s'adresse à certains aspects identitaires qui sont souvent à l'avant-scène quand on explore la transition vers une langue étrangère:

«Identité vaut pour immunité, l'une s'identifie à l'autre. L'étrangeté et l'étrangèreté deviennent communes et quotidiennes. Cela se traduit par une extériorisation du moi : il faut me mesurer, me tester, me contrôler» (Nancy, 2000, 33).

On apprend à vivre avec ce qui nous est étranger et même à s'identifier à cette étrangeté, dans la mesure où l'on parvient à modifier son système immunitaire.

C'est l'extériorisation des mots de la langue étrangère qui est à évaluer, à tester, ce qui risque d'être assez contrôlant et de menacer le sens d'appartenance. L'accent et le mauvais choix de mots font obstruction à la compréhension de l'autre. Ils exigent plusieurs tentatives pour s'exprimer, parfois sans succès, ce qui risque d'être aliénant. L'immigrant se vit comme n'appartenant ni à son pays d'origine ni à son pays d'accueil. La pratique auprès d'adolescents de familles immigrantes démontre que ceux-ci ont beaucoup de mal à vivre cette dualité. À ce sujet, un jeune homme d'origine guinéenne me confiait: «Je suis comme un chocolat: noir à l'extérieur et blanc à l'intérieur».

À Toronto, on me considérait comme une «frog» (c'est ainsi qu'on désignait les Français parce qu'ils mangent des cuisses de grenouille). À Montréal, ma ville natale, on disait que je venais du «ROC» (rest of Canada). J'ignorais ce que signifiait le ROC à cette époque. Plus de quarante ans plus tard, je répétais certaines expériences vécues à l'âge de dix ans: dans mon vocabulaire d'enfant, «sidéré» signifiait «avoir le sida» et «d'emblée» m'était un mot inconnu. Je parlais des «semaines de folle» au lieu de semaines de fou! Ma pensée et mes associations se formulaient en anglais. Lorsque le présentateur d'un des séminaires du mardi (à l'Institut de psychanalyse de Montréal) parlait avec insistance du pervers, j'entendais «le père vert», que j'associais à André Green. Lors d'un congrès international de psychanalyse au Chili où je parlais en espagnol, on me demanda si j'étais brésilienne car on se rendait compte que l'espagnol 
n'était pas ma langue d'origine. En fait, il existe dans mon monde interne deux «matrices», deux espaces à l'intérieur desquels s'effectue un aller-retour linguistique, ce qui ne mène pas à un rejet mais plutôt à un aller de moi à moi par le retour des mots. Cela dit, je partage parfois avec les immigrants un sentiment d'être étrangère, de ne pas vraiment appartenir à mon milieu.

À ce sujet, Nancy ajoute :

«L'intrus n'est pas un autre que moi-même et l'homme luimême. Pas un autre que le même qui n'en finit pas de s'altérer à la fois aiguisé et épuisé, dénudé et suréquipé, intrus dans le monde aussi bien qu'en soi-même, inquiétante poussée de l'étrange conatus d'une infinité excroissante.» (Nancy, 2000, 45)

Certains patients immigrés, plus carencés, évoquent le sentiment d'être contaminés, de ne pouvoir espérer créer de liens avec l'autre. D'autres, ayant vécu plusieurs immigrations, ainsi que l'apprentissage de plusieurs langues, apportent un témoignage différent.

Lors d'une conversation récente avec Naïm Kattan ${ }^{1}$, au cours de laquelle il abordait la question d'identité, il me confia que son identité était juive en premier lieu mais qu'en réalité, il avait eu trois naissances : à Bagdad, à Paris et à Montréal. Il m'expliqua que pour lui, l'identité n'est pas fixe mais toujours en mouvance.

\section{La violence nécessaire à l'apprentissage de la langue... D'une seconde langue?}

Que penser de l'obligation d'apprendre une autre langue? Cette obligation est en fait la deuxième. Piera Aulagnier parle de la violence primaire et nécessaire:

«Si [...] l'offre précède la demande, si le sein donné avant que la bouche sache que c'est de lui qu'elle est en attente, ce décalage est encore plus évident et plus total dans le registre du sens. La parole maternelle déverse un flux porteur et créateur de sens, qui anticipe de loin sur la capacité de l'infans d'en reconnaître la signification et de la reprendre à son compte.» (Aulagnier, 1975, 31)

«Nous appelons violence primaire, l'action psychique par laquelle on impose à la psyché d'un autre un choix, une pensée ou une action qui sont motivés par le désir de celui qui l'impose mais qui s'étayent sur un objet qui répond pour l'autre à la catégorie du nécessaire. En reliant le registre du désir de l'un à celui du besoin et du nécessaire de l'autre, la visée de la violence s'assure de sa victoire: la violence primaire, en instrumentant le 
désir sur l'objet d'un besoin, atteint son but qui est de faire de la réalisation du désir de celui qui l'exerce ce qui deviendra l'objet demandé par celui qui la subit. » (Aulagnier, 1975, 40)

L'apprentissage d'une deuxième langue, tout autant imposé que l'apprentissage de la première, risque d'être vécu comme une réédition de cette violence primaire. L'immigré qui se retrouve dans un nouveau pays baigne dans une nouvelle langue - bain sonore étranger qui semble faussé, qui heurte les oreilles, dont l'écoute ne permet pas l'appropriation d'un sens. Il se retrouve face à l'attente de l'autre et dans l'impossibilité d'y répondre. Les vignettes cliniques qui suivent illustrent le fait que l'apprentissage d'une nouvelle langue compte parmi les enjeux de séparation importants au niveau interpersonnel, comme au niveau d'une césure et d'une discrimination intrapsychique.

\section{Anita}

Anita, professionnelle d'origine sud-américaine, est venue au Canada, nous ditelle, parce que son conjoint souhaitait émigrer. Le couple, ayant abandonné la possibilité d'avoir un enfant à cause des problèmes de fécondité de madame, entrevoyait une nouvelle vie et nourrissait de nouveaux espoirs. Peu après son arrivée ici, Anita se retrouve enceinte. Elle vit une grossesse et un accouchement difficiles. Le lien avec son fils s'établit difficilement. Elle est à la fois heureuse d'avoir un enfant et déçue des changements que cela impose à ses projets de vie au Canada. Malgré son état dépressif, elle profite de l'accès à un centre de jour pour s'inscrire à des cours de francisation. Elle réussit bien les activités langagières écrites mais elle fait peu de progrès au niveau oral. Elle répète que pour elle, le français s'avère une langue impossible. Durant cette période, son père décède et elle ne peut voyager à cause de contraintes associées à son processus migratoire.

Je la rencontre au moment où son professeur lui impose un arrêt du cours de français parce qu'elle fait peu de progrès. Elle se sent persécutée, dévastée par cet échec, car elle est consciente de l'importance pour elle de l'acquisition du français, sans lequel son avenir professionnel est en péril. Or il se trouve que le professeur de français ressemble physiquement à sa mère. Anita la perçoit donc comme excessivement intrusive. À son insu, elle effectuait sur ce professeur un transfert négatif qui entravait l'apprentissage. Parallèlement à ces évènements, Anita reçoit l'autorisation de voyager, de retourner dans son pays pour faire le deuil de son père. Elle me confie qu'elle ne souhaite pas revenir, qu'elle quitte avec son enfant, et laisse son mari ici. Son ambivalence face à ce souhait évoque la relation fusionnelle et intrusive qu'elle a vécue avec sa mère. Elle s'inquiète d'ailleurs de cette rencontre prochaine avec cette dernière, qu'elle n'a pas vue depuis deux ans. Nous explorons ses inquiétudes face à cette relation et évoquons l'hypothèse selon laquelle l'immigration et l'apprentissage du français représenteraient pour elle une coupure, une différentiation significative avec sa mère. 
Quatre mois plus tard, elle rentre à Montréal, s'inscrit à une nouvelle école de langues. À l'intérieur de six mois, non seulement elle parle français d'une façon plus qu'adéquate, mais elle a trouvé un travail qui lui plaît. La maîtrise de la nouvelle langue se fit à la suite de changements de perception de l'image maternelle et peut-être même de réorganisation d'évènements inconscients. Elle explique qu'en rentrant dans son pays elle a compris que sa décision d'immigrer était tout autant la sienne que celle de son conjoint, et que maintenant elle pouvait s'autoriser à avoir des désirs autres que ceux de sa mère. Le lien maternel ambivalent et fusionnel lui interdisait l'apprentissage de la langue de son pays d'accueil. La nouvelle langue a pu naître sous le signe de la reconnaissance de la séparation. Nous comprenons davantage l'impact qu'a pu avoir ce premier professeur qui évoquait chez elle des souvenirs très intenses de sa mère. Le français devint sa langue de «femme» et remplaça celle de la petite fille indifférenciée de sa mère. Elle pouvait maintenant se permettre d'extérioriser la connaissance de sa nouvelle langue, de même que son statut de femme. Comme le dit Biancotti :

«Parfois les parcours internes à travers les langues conduisent vers l'intégration et la récupération du contact avec des parties de soi négligées et éloignées dans le passé.» (Biancotti dans Amati Mehler et al., 1994, 217)

Le retour au pays lui a permis de résoudre des aspects de sa relation ambivalente à l'image maternelle mais je crois que l'immigration, cette césure avec la mère, lui a permis de devenir enceinte une fois arrivée au Québec malgré les problèmes d'infertilité diagnostiqués dans son pays.

\section{Jena}

Quel contraste avec Jena, qui grandit en Argentine issue de parents de l'Europe de l'Est, qui n'est exposée qu'au russe jusqu'à l'âge de trois ans, âge auquel elle entre en garderie où l'on parle espagnol. Elle apprend cette nouvelle langue rapidement car le russe est désormais banni à la maison. À six ans, elle est inscrite au lycée français et toute sa scolarisation se fait dans cette langue. Jeune adulte, elle émigre en France et ensuite au Québec. Lors d'un voyage en Russie, on lui dit qu'elle parle le russe avec un accent ukrainien. Sa grand-mère maternelle était ukrainienne : l'ukrainien avait été interdit chez elle par sa mère, qui avait honte des origines paysannes de sa propre mère. Tout comme sa mère, Jena renonce à ses origines et le français devient pour elle la langue qui lui permet une ouverture, une issue, une re-création, une liberté. En plus, le français représente une langue neutre a-conflictuelle qui n'est pas la langue des conflits vécus entre ses parents. Le changement de langue a été mis au service de la fuite des conflits originaires en permettant une distanciation avec les mots de la langue d'origine — des mots chargés émotionnellement et sensoriellement. 
Pour revenir à l'utilisation et à l'apprentissage d'une langue autre que la langue maternelle, les exemples ci-dessus suscitent quelques questions : quel est le rôle du lien avec la mère dans le désir ou le non-désir d'apprendre une autre langue? Qu'est-ce qui est transmis d'une génération à l'autre face au rejet de sa langue maternelle et que représente le choix de la langue d'adoption? Comment se vit l'imposition d'apprendre une autre langue?

En ce qui concerne l'immigration, il n'y a pas de choix. Il faut apprendre la langue pour survivre. Anita était incapable d'utiliser sa connaissance du français avant qu'elle ne se sépare du lien fusionnel avec sa mère. L'apprentissage lui était partiellement interdit.

Avec Jena par contre, il y aurait eu une transmission, une répétition de l'interdit. Chez sa mère l'interdit était de parler l'ukrainien tandis que chez elle l'interdit était de parler le russe. Elle a rejeté la langue adoptée par sa mère (l'espagnol) pour choisir le français comme la langue dans laquelle elle travaille, pense, rêve. Ses liens avec sa mère, comme ceux de celle-ci avec sa propre mère, sont fracturés et pénibles.

\section{La nouvelle langue : une liberté ?}

Le récit de Jena ci-dessus évoque les commentaires de Atik Rahimi lors d'une entrevue le 10 novembre sur RFI (Radio France Internationale) au moment où l'on annonçait qu'il avait remporté prix Goncourt 2008. D'origine afghane, Rahimi souligne qu'il n'aurait pu écrire son dernier livre, Syngué sabour dans sa langue maternelle, le persan.

«C'est une chose mystérieuse, étrange, mais la langue maternelle impose mille et une contraintes. C'est avec la langue maternelle que vous connaissez vos interdits, vos tabous, vos limites. Mais, la langue d'adoption, c'est la langue de liberté ». On lit dans le journal Le Devoir du samedi 16 novembre rapportant ses propos : «[...] il n'aurait pu l'écrire en persan, insiste-t-il [...] je me serais trop censuré. C'est la langue dans laquelle j'ai grandi, dans laquelle j'ai été empêché de prononcer certains mots, et, quoique je fasse, mon inconscient m'interdit de les prononcer. »

Parlant de son choix de surtout écrire en français, Naim Kattan me confie: «Quand j'écris en français je me sens comme quelqu'un qui obéit, qui est soumis, comme je dois l'être aux règles de grammaire et de syntaxe... en effet je suis comme un serviteur».

Atik Rahimi parle dans la langue tandis que Naïm Kattan fait parler la langue tout en se laissant porter. J'ose penser que Naim Kattan et Atik Rahimi ont une relation semblable à leur langue d'adoption, c'est-à-dire un sentiment de liberté, un encadrement permettant plus d'ouverture.

La langue maternelle de mes deux parents était le français. Tous deux avaient appris l'anglais à l'école. Sauf mon grand-père maternel, qui a appris un peu d'anglais tardivement, mes grands-parents ne parlaient pas l'anglais. Malgré mes souvenirs assez vifs des commentaires de mes deux parents sur les maudits 
Anglais - malpropres et injustement avantagés dans le milieu du travail... car c'étaient les Anglais qui avaient droit aux promotions dans le moulin à papier du CIP (Canadian International Paper Company) — on m'impose une rupture avec ma langue à l'âge de dix ans, rupture qui a perduré pendant plus de 40 ans. Jusqu'à ce jour, l'anglais reste la langue d'échange avec ma mère.

Ma mère privilégiait l'anglais. À son insu, elle répétait avec moi ce qu'elle avait vécu: la séparation du milieu familial et l'apprentissage de l'anglais. Mais pourquoi cette insistance à parler anglais ? On pourrait penser à une identification à l'agresseur: un rejet de la langue de la mère associé à la fragilité du lien maternel, rejet que j'ai aussi transmis à ma fille polyglotte qui parle le français avec un accent anglais prononcé. Il y a là trois générations de femmes pour lesquelles la langue maternelle est en souffrance et qui ont choisi d'autres langues pour s'exprimer, voire pour s'identifier.

Certains de ces exemples mettent en évidence la liberté qui accompagne le choix d'une nouvelle langue. Pour Anita, la nouvelle langue représente la possibilité de devenir un sujet, une femme adulte. Pour Jena, le français représente la possibilité de se ré-inventer dans une langue a-conflictuelle. Pour moi, c'est l'apprentissage de l'espagnol qui a été vécu comme une source de plaisir, de découvertes et d'ouverture.

Les vieux psychiatres traitaient leurs patients schizophrènes en les incitant à apprendre une autre langue, comme si la folie ne se transmettait pas dans la nouvelle langue... comme s'ils croyaient pouvoir reconfigurer le « disque dur».

\section{Le non-désir d'apprendre une autre langue, ou le refus de la parler}

Considérant le désir ou le non-désir d'apprendre une autre langue hors d'un contexte migratoire, une collègue polyglotte faisait un parallèle avec le fait que certains enfants souhaitent goûter à tout tandis que d'autres «ne mangent que du beurre d'arachides ». Bien que tenant compte du contexte politique et culturel, je crois que la surdétermination de l'être humain nous permet quand même de poser certaines questions : pourquoi certains anglophones montréalais parlent-ils français tandis que d'autres n'en connaissent pas un mot et ont une aversion à l'apprendre? Comment comprendre les Pieds noirs, bien installés au Maghreb, qui envoient leurs enfants au lycée français et qui ne les exposent pas à l'apprentissage de l'arabe?

Quel contraste avec ma jeune patiente infirmière du Bas-du-Fleuve qui s'est rendue en Californie pendant six mois dans le but d'apprendre l'anglais. Elle a aussi choisi d'aller étudier à McGill en anglais, au lieu de fréquenter l'Université de Montréal, ce qui aurait été beaucoup plus facile pour elle. Très tôt dans nos rencontres, j'apprends qu'elle avait eu beaucoup de mal à effectuer la séparation avec ses parents, surtout avec sa mère. À 20 ans, elle vivait toujours avec eux. Malgré son statut de jeune adulte, ses parents continuaient à avoir beaucoup d'influence sur ses choix et sur sa vie quotidienne. Le choix d'une langue et de vécus étrangers lui permettait de créer un espace où elle pouvait redéfinir son identité. 
Comment comprendre ceux qui n'éprouvent aucun désir d'apprendre la langue de l'autre ? Peut-être qu'il s'agit d'un manque de curiosité, d'une haine, d'un malaise avec l'étranger, d'une crainte de perte identitaire (en contraste avec une fuite identitaire) ou encore d'un déni du manque — un sentiment de complétude illusoire. Et que dire de ceux qui se lancent avec avidité dans l'apprentissage de la langue de l'autre? La pratique de plusieurs langues serait-elle une façon de pallier le manque? Le fait de nier sa langue maternelle en apprenant avec avidité une autre langue pourrait aussi être une tentative d'effacer un manque dans certaines situations.

Le manque, la mère, et la langue me renvoient au texte d'Abraham et Torok, L'écorce et le noyau:

«Le passage de la bouche pleine de sein à la bouche pleine de mots s'effectue au travers d'expériences de bouche vide. Apprendre à remplir de mots le vide de la bouche, voilà un premier paradigme de l'introjection. On comprend qu'elle ne peut s'opérer qu'avec l'assistance constante d'une mère, possédant elle-même le langage. Sa constance [...] est le garant nécessaire de la signification des mots. Lorsque cette garantie est acquise, mais alors seulement, les mots peuvent remplacer la présence maternelle et donner lieu à de nouvelles introjections. » (Abraham et Torok, 1987, 263)

L'apprentissage d'une nouvelle langue pourrait-il se comparer à l'introjection? S'agit-il de remplir de nouveaux mots le vide de la bouche? Je crois qu'il existe une relation entre les liens avec la mère et l'apprentissage d'une langue étrangère ou le désir d'apprendre une langue étrangère La bouche, toujours remplie par le sein, aurait du mal à faire de la place à de nouveaux mots. Cela évoque la relation avec des parents intrusifs ou des parents qui craignent une perte identitaire.
«D'abord la bouche vide, puis l'absence des objets deviennent parole, enfin les expériences des mots elles-mêmes se convertissent en d'autres mots. Ainsi le vide oral originel aura- t-il trouvé remède à tous ses manques par leur conversion en rapport de langage avec la communauté parlante» (Abraham et Torok, 1987, 263)

Quand on parvient à «introjecter» une langue qui n'est pas la sienne et qu'on arrive à se sentir comme étranger à sa propre langue, n'arrive-t-on pas à dire : «La langue que je parle n'est plus la mienne et la mienne ne m'habite plus » Le succès de l'introjection ne serait-il pas accompagné par de vifs sentiments inconscients de perte?

De nos jours, dans les milieux urbains, que ce soit à la suite d'immigration, de l'imposition de parler une autre langue, d'une interdiction de parler sa langue ou 
simplement de l'effet osmotique d'autres cultures et d'autres langues, plusieurs risquent de se sentir comme s'ils vivaient à l'extérieur de la communauté parlante car ils ne comprennent pas la langue du milieu dans lequel ils se retrouvent. Pour certains, la membrane linguistique perd sa caractéristique d'étanchéité qui lui permettait de ne contenir que les sons familiers. Maintenant, elle doit devenir perméable ou même poreuse et permettre l'accès aux nouveaux sons et mots. Récemment, lors d'une fête, nous étions dix personnes d'origines diverses autour d'une table: israélienne, argentine, mexicaine, tchèque, polonaise, grecque, américaine, française et québécoise. La première question fut «quelle langue allons-nous parler?» Ce soir- là le français gagna, étant la langue partagée par la majorité. Cette hétérogénéité de plus en plus fréquente ne risque- t-elle pas d'être vécue comme une perte de frontières, de limites et d'identité ? Cette incapacité à tolérer la perte, la lacune, pourrait conduire à quelque chose qui ressemble au fantasme d'incorporation décrit par Abraham et Torok?

Abraham et Torok soulignent que les obstacles à l'introjection mènent à l'incorporation:

«Si tout fantasme est refus d'introjecter et négation d'une lacune, on devra se demander $[\ldots]$ pourquoi leur contenu vise-t-il la métaphore même de l'introjection? [...] En effet, pour que l'incorporation procède de manière à accomplir à la lettre la métaphore de l'introjection, il faut que le processus, habituellement spontané, devienne l'objet d'une thématisation, d'un traitement réflexif en quelque sorte. Or, cela doit advenir dans un seul cas: lorsque le travail d'introjection à peine commencé ou entrevu se heurte à un obstacle prohibitif. Ceci posé, il nous est loisible de préciser le lieu de cet obstacle : il se trouve, de toute évidence, dans la bouche même où siègent les phénomènes présidant à l'introjection. C'est parce que la bouche ne peut pas articuler certains mots, énoncer certaines phrases-pour des raisons à déterminer - que l'on y prendra en fantasme, l'innommable, la chose elle-même. Le vide de la bouche appelant en vain, pour se remplir, des paroles introjectives, redevient la bouche avide de nourriture d'avant la parole: à défaut de pouvoir se nourrir des mots qui s'échangent avec autrui, elle va s'introduire, fantasmatiquement, tout ou partie d'une personne, seule dépositaire de ce qui n'a pas de nom. Depuis l'introjection, avérée impossible, le passage décisif à l'incorporation s'effectue donc au moment où les mots de la bouche ne venant pas combler le vide du sujet, celui-ci y introduit une chose imaginaire.» (Abraham, Torok, 1987, 264)

Cette impossibilité à combler la lacune des mots, de parler à quelqu'un des manques, laisse la place à l'imaginaire et à l'illusion. Le refus d'apprendre une 
autre langue en gardant la bouche pleine de façon illusoire, l'incapacité à tolérer la bouche vide qui permettrait aux nouveaux mots d'avoir un espace: ce refus d'apprentissage serait-il lié à un refus de perdre, à un désir non avouable, tout comme une «incorporation»?

\section{Le narcissisme des petites différences}

Le discours de mes collègues fait écho aux dires de mon enfance: les maudits Anglais, les juifs dont il fallait se méfier, qui avaient accès au pouvoir, au succès et à l'argent. Peut-être que ces fantasmes persécuteurs continuent à faire obstacle à l'apprentissage de la langue de l'autre tout comme le fantasme de l'incorporation fait obstacle à l'introjection?

Un jeune patient anglophone de 22 ans, né à Montréal, enragé quand on s'adresse à lui en français, me fait part de son enfance au cours de laquelle il aurait vécu des sévices à l'école. Il était souvent l'objet de blagues, de jurons, d'attaques physiques sur la route du retour après l'école et d'insultes («tête carrée»). Une collègue anglophone me disait que ce n'est que récemment qu'elle éprouve un désir d'apprendre le français. Elle aurait souffert dans son voisinage et dans son école française où elle était l'une des seules anglophones. Elle ne comprenait pas le français, se faisait taquiner et vivait un grand isolement dû à son étrangeté et à son incapacité de s'intégrer. Ce genre de récits est tout autant présent dans le milieu francophone et évoque le narcissisme des petites différences tel que le décrit Freud dans Malaise dans la Civilisation (1930):

«Il est toujours possible d'unir les uns aux autres par les liens de l'amour une plus grande masse d'hommes à la seule condition qu'il en reste d'autres en dehors d'elle pour recevoir les coups. Je me suis occupé jadis de ce phénomène que justement les communautés voisines et même apparentées se combattent et se raillent réciproquement; par exemple les Espagnols et les Portugais, les Anglais et les Écossais» (Freud, 1930, 56)

Observation qui pourrait bien concerner les francophones et les anglophones de Montréal, de même que les différents regroupements psychanalytiques. Des relations haineuses, méprisantes se sont établies entre les francophones et les anglophones dans une situation où les rapprochements s'avèrent chatouilleux, complexes et peut-être indésirables. L'enjeu se complique quand on parle des différents groupes psychanalytiques car, en plus de la langue parlée, le paradigme psychanalytique comme tel accuse des différences tout aussi significatives. Je soulignerai qu'il y a peut-être autant de relations haineuses entre les selfpsychologists, les lacaniens et les freudiens dans une ville comme Toronto - où la langue partagée est l'anglais. 


\section{La langue dans ses deux sens}

En 2008, lors d'une visite au musée d'Art Moderne de Barcelone, je fus bouleversée par l'exposition de Susan Spero, une artiste féministe d'origine Américaine. L'exposition intitulée « la langue », abordait le thème de la dislocation linguistique. Une de ses œuvres que je trouvais particulièrement dérangeante représentait la grande tête engorgée du Codex Artaud XVII avec sa langue protubérante d'apparence phallique qui cachait partiellement un texte en filigrane. Dans sa forme primitive, anatomique, cette langue réclamait l'espace de la langue écrite et disait «L'obscène pesanteur phallique d'une langue qui prie». Cette langue, qui est corps et langage, qui émane du corps, parle du corps. Aussi, comme l'écrit Kristeva :

«La langue qui agit comme un organe public et privé, le sens et le langage mais aussi celui de la sensualité, celle qui lèche goûte et fouille.» (dans Edward Scheer, ed. Antonin Artaud, 1972, 119).

Lucy Bradock, (2005) dans son essai inédit sur le travail de Susan Spero, associe prays (prie), pries (soulève), et preys upon (être prédateur) ce qui exprime particulièrement bien les propos de Spero, qui luttait pour exprimer le féminin dans un contexte visuel et textuel, dominé par ce qu'elle appelle le phallogocentrisme. Pour certains québécois, la langue anglaise serait-elle la langue phallique? Un collègue, à qui je décrivais ce tableau, me soulignait comment cette langue/pénis qui sortait de la bouche était l'envers d'une fellation. Dans ce contexte, une fellation non-désirée, tout comme la langue «phallique » indésirable, doit être évitée.

Cette langue a pendant si longtemps pour moi recouvert le texte français jusqu'au point de l'occulter, tout comme dans le tableau de Spero. Y aurait-il un parallèle à faire avec la lutte féministe pour avoir sa propre voix (langue) et ne pas permettre l'intrusion d'autres langues? La lutte pour un «monolingue», le refus d'apprendre et/ou de transiger dans une autre langue et de subir les pertes qui accompagneraient cette incursion seraient-ils une protection? Une défense? L'évitement de la langue phallique serait-il une façon de discriminer, de séparer, de différencier et de maintenir sa langue pour se retrouver et pour se réunifier? Par contre, l'autre langue, cette langue qui recouvre le texte originel, pourrait-elle permettre de trouver des mots pour dire ce qu'on ne peut pas dire ou comprendre dans notre langue maternelle?

«Du français à l'anglais se créent de nouvelles transmissions de symbolisations et de significations dans un réseau mobile de clivages et de connexions. » (Amati Mehler et al., 1994, 98)

L'anglais est vécu comme la langue de l'argent, du pouvoir, de la pensée logique, voire de la masculinité. L'adoption de cette langue «phallique » serait-elle 
liée à un évitement de la féminité pour certaines personnes, à une désidentification avec la mère, à un refus de se renfermer dans un lien fusionnel ? Le français nous oblige à identifier le masculin et le féminin tandis que l'anglais est beaucoup plus neutre. Je remarque que l'on parle de «la Société psychanalytique de Montréal» tandis qu'on dit «le Quebec English Psychoanalytic Society» pour désigner nos collègues anglophones.

\section{La thérapie et la langue}

La confrontation avec la langue de l'autre, au sens de langue d'ailleurs, n'épargne aucun thérapeute travaillant en milieu urbain. Même quand on parle la même langue, on ne parle pas la même langue. Par ailleurs, est-ce qu'on ne parle pas toujours la langue de l'autre? L'intra langue risque d'être tout aussi difficile que l'inter langue. Les différences entre les niveaux sociaux et régionaux sont importantes. Cela me rappelle certains souvenirs d'enfance où je ne parlais pas le même français qu'aujourd'hui: on disait «moé et toé» et mon grand-père me demandait d'aller «qu'ri sa pipe». Cela évoque aussi une séance avec la patiente d'origine québécoise qui me dit que «son chum faisait ses études médicales» ce que je lui relance pour apprendre que son copain était cobaye pour le compte d'une compagnie pharmaceutique.

Comment penser la thérapie ou l'analyse quand les deux partenaires ne partagent pas la même langue maternelle? Qu'est-ce qui motive le choix de l'analyste (homme ou femme) et de la langue des séances ? Le choix d'une langue ne peut être laissé au hasard car il peut servir les défenses psychiques et ne pas permettre à l'association de suivre son libre cours.

Certains éléments risquent d'être occultés, de demeurer fermés quand l'analyste et l'analysand partagent ce processus dans une langue qui n'est pas la leur. Bien sûr, il y a toujours des points aveugles et on pourrait même suggérer que l'utilisation d'une langue partagée, sans être la langue maternelle, risque de conduire à des découvertes inusitées. On peut aussi penser que l'inconscient fait référence à une langue universelle. L'accès à l'inconscient se fait par la langue : les lapsus, les récits de rêves, les fantasmes et tous ces moments où l'on est parlé, où les mots sortent malgré nous. Les décalages entre la langue de l'analyste et celle de l'analysant risquent de compliquer la situation en fermant l'accès à certains secteurs psychiques. L'accès à l'inconscient, déjà difficile, serait sans doute plus facile dans sa propre langue. Le rêve qui passe par l'image exige le vocabulaire pour le décrire; il exige des paroles pleines, pas seulement des étiquettes.

Quel serait l'impact sur le transfert du fait que l'analyse n'ait pas lieu dans la langue maternelle? De prime abord, pourquoi choisit-on un analyste qui parle ou ne parle pas notre langue maternelle? J'apporterai mon propre vécu comme réponse partielle à cette question.

Ma première analyse se déroula en anglais avec un sud américain qui le parlait bien mais avec un accent espagnol. En écrivant ce texte, j'ai réalisé que je l'avais choisi parce qu'il parlait l'espagnol, cette langue que j'avais toujours souhaité 
apprendre au moment où on m'imposait l'anglais, et aussi, à mon insu, parce qu'il évoquait ma toute première relation amoureuse avec un hispanophone. Il y eut quelques échanges en espagnol durant l'analyse, mais j'imaginais un refus de sa part d'accepter mon discours en espagnol, sans doute lié aux aspects défensifs que ce changement de langue représentait.

Tout de même, c'est grâce à cette analyse que les aspects identitaires, de racines de filiation furent soulevés. Ayant été acceptée comme candidate pour la formation psychanalytique simultanément à Toronto et à Montréal, j’hésitais en pensant aux coûts de la navette hebdomadaire entre Toronto et Montréal, à la perte inévitable de plus d'une journée de travail par semaine, mais surtout à l'angoisse de me confronter à l'étrangeté de ma propre langue. J'avais adopté l'anglais et ma propre langue m'avait oubliée. Je me savais incapable d'écrire des rapports, des textes en français. La grammaire française de ma sixième année s'était fossilisée; depuis plus de quarante ans je n'entendais, ne lisais et ne parlais presque exclusivement que l'anglais. En plus de vivre une carence au niveau linguistique, j'étais dans un état de déculturation aigu. À l'étonnement de mes collègues, les personnages et les auteurs comme Vigneault, Marie- Claire Blais, Gabrielle Roy et des groupes comme Harmonium m'étaient inconnus. Je me suis laissé séduire par l'accueil chaleureux à la SPM lors du colloque de ce Printemps 1998, axé sur une présentation de Jean Imbeault. La passion, l'investissement des participants et la qualité des présentations m'avaient fascinée. Quand on change de langue, on change de contexte: je venais d'en vivre la preuve.

Le travail identitaire déjà entamé dans l'analyse m'avait permis de réfléchir et de ne pas suivre mon premier réflexe qui était de faire ma formation à Toronto.

Une fois ma formation terminée et après mon installation à Montréal, j'ai décidé de faire une tranche d'analyse, cette fois dans ma langue maternelle avec une analyste québécoise. Malgré mes quatre années de formation, j'avais du mal à m'exprimer en français au début de l'analyse et j'avais souvent recours à l'anglais sans savoir si mon analyste le comprenait ou non. Au fil du temps, le désir, l'exigence et l'espace dont je disposais pour m'exprimer en français me permirent d'avoir accès à des vécus davantage archaïques et pulsionnels. Je crois que le nouveau matériel qui a émergé durant cette analyse n'aurait jamais vu le jour dans l'analyse précédente. Je crois qu'il y a un impact transférentiel quand l'analyse a lieu dans la langue maternelle et aussi dans une autre langue psychanalytique, par exemple: les différences entre un didacticien d'origine sud-américaine avec des références kleiniennes, freudiennes, bioniennes, lacaniennes, et une analyste habilitée formée à la SPM et sans doute influencée par Freud, Green, Winnicott, Khan, Shaeffer, Gantheret, Pontalis, Aulagnier etc. Le contexte torontois anglophone est saturé de la psychologie du Self, de l'intersubjectivité et de la relation à l'objet tandis que le milieu montréalais lui, parle surtout de pulsions et de sexualité. Il faut ajouter à ces différences les modalités d'intervention: l'une est davantage axée sur le concret et sur le non-verbal tandis que l'autre tient plus au symbolique. 
D'origine polonaise, Eva Hoffman (dans Amati Mehler et al., 1994, 240) parvenait à décrire ses vécus en anglais et croyait que la langue acquise à une certaine distance de l'enfance arrivait parfois à exprimer le monde interne avec la même fidélité que la langue maternelle, y compris par rapport aux expériences primordiales, ce qui n'est conforme ni à mon vécu ni à mes expériences cliniques.

Le patient sud américain qui bascule soudainement vers l'espagnol sous l'influence d'un tsunami affectif, démontrerait-il qu'on a plus facilement accès à l'inconscient dans notre langue maternelle? La réaction affective qui accompagne le changement de langue est-elle provoquée par l'effort que lui impose sa langue adoptive, ou est-ce l'étrangeté de l'autre langue qui s'allie avec l'étrangeté de l'inconscient, tout autant qu'avec les pulsions et les imagos archaïques qui ont laissé leurs traces dans la langue maternelle?

D'après Benjamin Lee Whorf :

«La structure de la langue détermine la perception de l'individu et l'organisation de sa pensée » et «les catégories fondamentales de la pensée ne sont pas les mêmes dans les différentes langues (temps, espace, sujet. Objet). Chaque groupe de langues aura donc une étroite correspondance avec certaines représentations du monde même, ou, en sera la cause.» (dans Amati Mehler et al., 1994, 231)

Ces propos évoquent la langue arabe qui contient une multitude de mots pour décrire le sable. Ou encore dans la langue inuit où existent plus de trente mots pour décrire la neige.

On me fait souvent le commentaire que je ne suis pas la même en français et en anglais, comme si la langue parlée affectait aussi la perception de l'autre à mon égard.

D'autres, (comme Chomsky), sont en désaccord avec ce genre de commentaire, prônant plutôt le caractère inné des universaux linguistiques :

«Il y aurait une propriété commune de langage qui se révèle en toutes langues ce qui assurerait la possibilité de dépasser les barrières linguistiques imposées par la diversité des langues parlées dans le monde. » (dans Amati Mehler et al., 1994, 233)

Dans son récit de la cure d'Anna O., Freud (1892) décrit comment, suite à une période de mutisme, celle-ci ne pouvait parler et écrire qu'en anglais. Par ailleurs, elle comprenait toujours sa langue d'origine, l'Allemand. L'impossibilité de parler allemand était liée à sa mère et à l'ambivalence de ses expériences orales.

$\mathrm{Y}$ aurait-il davantage de lapsus quand on utilise une seconde langue ? Je pense à la patiente bilingue, qui avait longtemps délaissé sa langue maternelle, le français. En parlant de sa relation avec son père elle me dit: «j'étais son 
partenaire ». En premier lieu elle résiste à l'attention portée par l'analyste à ce lapsus, prétendant qu'il ne s'agissait que d'une erreur linguistique. Mais bientôt elle l'associe à un fantasme révélé quelques mois auparavant «si j'avais été un homme». Le lapsus mettait en scène le fantasme déjà évoqué et supprimé de nouveau. Le refoulement et les clivages peuvent utiliser les différentes langues comme véhicules.

Perd-on du sens quand on passe d'une langue à l'autre? L'analyste d'une autre langue maternelle que son analysant aurait-il moins de distractions qu'il n'en aurait dans sa propre langue? Biancotti souligne que :

«Le bilinguisme nous oblige à mentir. En nommant quelque chose on est conscient des altérations que la chose même subit selon la langue même qui la nomme. Oiseau est tiède, luisant, lisse: pajaro- part comme une flèche» Amati Mehler et al., 1994, 206)

Il y a aussi l'exemple classique de Lévi-Strauss entre «fromage» et «cheese». J'ajouterais l'impact des mots : angoisse et angustia (espagnol) se traduisent tous deux en anglais par anxiety, mais n'évoquent aucunement le même sens et surtout pas le même vécu affectif. On trouve un autre exemple frappant dans la différence entre pulsion et drive. Cela explique peut-être ce que ressentent mes collègues quand ils me disent différente selon la langue que j'utilise. Il n'existe pas deux langues assez semblables pour qu' on leur fasse représenter affectivement la même chose malgré l'utilisation des bonnes étiquettes. Selon Biancotti, cela ne constitue pas une richesse mais plutôt un égarement et parfois même une perte de réalité.

Mon premier cas contrôle était une patiente anglophone. Mon superviseur insistait pour ce que je lui fasse rapport des séances en anglais, ce qui me soulageait parce que j'avais beaucoup de mal à m'exprimer en français. Avec un autre superviseur pour un autre cas anglophone, la langue d'échange était le français sauf quand la traduction s'avérait trop déroutante. Il est essentiel que le contrôleur comprenne la langue du patient, affirme Jacques Mauger, didacticien à la SPM, «après tout la psychanalyse est une cure par la parole. Ne miser que sur le contenu serait comme passer à côté de la plaque». Il affirme que s'il ne comprend pas la langue du patient dont il est question, il réfère le candidat à un contrôleur qui, lui, la comprendra.

Mon deuxième cas contrôle, qui s'avérait mon premier cas en français, coïncidait avec la période où je commençais à me réapproprier le français. Le discours du patient suscitait en moi des images dont je ne parvenais pas à penser dans la langue du patient mais plutôt en anglais. La plupart du temps, je parvenais, sans trop m'en apercevoir à les traduire dans la langue du patient. Tout de même, j'étais consciente de l'effort requis pour répondre en français et pour traduire mes pensées.

Mon patient racontait des rêves chargés de fantasmes homosexuels. Lors d'une séance, il me parlait «d'une personne, elle» alors qu'il me parlait d'un homme. Je 
pensais qu'il avait fait un lapsus et qu'il aurait dû dire «il» ce que je lui soulignai. Il m'expliqua qu'en français «personne» est féminin. Le client a interrompu son analyse après cette séance et c'est seulement en écrivant ce texte que je me suis remémoré cet événement ainsi que le malentendu linguistique et son impact sur le patient. On peut bien sûr attribuer cet échec à l'inexpérience de l'analyste qui était convaincue que son patient était homosexuel et faute d'une attention flottante, cherchait à confirmer son hypothèse.

Une des histoires qui m'a le plus frappée concernant la langue est celle d'une collègue qui venait de l'Europe de l'Est et qui parlait à peine l'anglais. Sa formation exigeait qu'elle fît une analyse et elle fut référée à un candidat pour qui elle fut un cas contrôle. Le candidat ne tarda pas à lui proposer de s'allonger sur son divan. Quelques mois plus tard, elle décompensait, était angoissée et morcelée. Le manque de mots pour s'exprimer et la privation imposée par le divan lui étaient insupportables. L'analyse se termina abruptement et elle fut prise en charge par un analyste senior qui la vit en face à face. Le désir du cas contrôle avait pris le dessus dans la pensée du candidat et du contrôleur: ils avaient escamoté l'importance d'une parole qui doit pouvoir être exprimée et entendue dans une analyse.

Ces thèmes méritent davantage d'élaboration que ce que j'ai pu leur accorder dans ces quelques pages. Néanmoins, je crois qu'il s'avère de plus en plus essentiel de leur prêter attention. Après tout, quels analystes ou thérapeutes ne sont pas en contact avec des patients dont la langue maternelle est différente de la leur? Ne sommes-nous pas toujours en double traduction? L'analyste traduit une langue que tous les deux parlent mais que ni l'un ni l'autre connaissent.

carole levaque 4878 rue hutchison montréal, qc $\mathrm{h} 2 \mathrm{v} 4 \mathrm{a} 3$

\section{Note}

1. Naïm Kattan est né à Bagdad et a immigré au Québec en 1954. Il est romancier, essayiste et critique littéraire. Il n'a jamais publié de livres dans sa langue, l'arabe. Il a écrit tous ses livres en français. À 80 ans, il publiera bientôt son quarantième livre. Dans plusieurs situations l'évolution identitaire se vit comme un phénomène qui permet une liberté et une croissance plutôt qu'une césure.

\section{Références}

Abraham, N., et Torok, M., 1978, L'écorce et le noyau, Flammarion, 1987.

Aulagnier, P., 1975, La violence de l'interprétation, Presses Universitaires de France, 1995.

Amati Mehler, J., Argentieri, S., et Canestri, J., 1994, La Babel de l'inconscient, Presses universitaires de France.

Derrida, J., 1996, Le monolinguisme de l'autre, Paris, Galilée

Freud, S., 1895, Études sur l'hystérie, Paris, PUF, 1956.

Freud, S, 1929, Le malaise dans la culture, Paris, PUF, 1995.

Danger! Le photocopillage détruit les revues. 
Green, A., et al., 1983, Langages. Deuxièmes rencontres psychanalytiques d'Aix-en-Provencce, in Le langage de la psychanalyse, Paris, Les Belles Lettres, 1984.

Kristeva, J., 2004, The subject in process, in Edward Scheer, ed, Antonin Artaud, A Critical Reader, London and New York, 119.

Nancy, J-L., 2000, L'intrus, Paris, Galilée. 